\title{
White matter lesions on magnetic resonance imaging in dementia with Lewy bodies, Alzheimer's disease, vascular dementia, and normal aging
}

\author{
R Barber, P Scheltens, A Gholkar, C Ballard, I McKeith, P Ince, R Perry, J O’Brien
}

Institute for the Health of the Elderly

R Barber

A Gholkar

I McKeith

R Perry

J O'Brien

MRC Neurochemical Pathology Unit, Newcastle General Hospital, Newcastle upon Tyne, UK C Ballard

$\mathrm{P}$ Ince

Department of Neurology,

Academisch

Ziekenhuis VU, Amsterdam, The Netherlands

P Scheltens

Correspondence to: Dr R Barber, Institute for the Health of the Elderly,

Newcastle General Hospital, Westgate Road, Newcastle upon Tyne, NE4 6BE, U.K. $\mathrm{Tel}+(0) 1912563274$ ext. 22986; fax +(0) 191219 5048; email

Robert.Barber@ncl.ac.uk

Received 26 August 1998 and in revised form 10 February 1999

Accepted 11 February 1999

\begin{abstract}
Objectives-Alzheimer's disease and vascular dementia are associated with an increase in changes in white matter on MRI. The aims were to investigate whether white matter changes also occur in dementia with Lewy bodies and to examine the relation between white matter lesions and the cognitive and noncognitive features of dementia with Lewy bodies, Alzheimer's disease, and vascular dementia.

Methods-Proton density and T2 weighted images were obtained on a 1.0 Tesla MRI scanner in patients with dementia with Lewy bodies (consensus criteria; $\mathbf{n}=27$, mean age $=75.9$ years), Alzheimer's disease

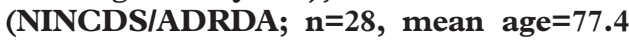
years), vascular dementia (NINDS/AIREN; $\mathrm{n}=25$, mean age $=76.8$ years), and normal controls $(n=26$, mean age $=76.2$ years $)$. Cognitive function, depressive symptoms, and psychotic features were assessed using a standardised protocol. Periventricular hyperintensities (PVHs), white matter hyperintensities (WMHs) and basal ganglia hyperintensities (BGHs) were visually rated blind to diagnosis using a semiquantitative scale.
\end{abstract}

Results-Periventricular hyperintensities were positively correlated with age and were more severe in all dementia groups than controls. Total deep hyperintensities scores (WMHs plus BGHs) were significantly higher in all dementia groups than controls and higher in patients with vascular dementia than those with dementia with Lewy bodies or Alzheimer's disease. In all patients with dementia, frontal WMHs were associated with higher depression scores and occipital WMHs were associated with an absence of visual hallucinations and delusions.

Conclusion-In common with Alzheimer's disease and vascular dementia, PVHs and WMHs were significantly more extensive in dementia with Lewy bodies than in controls. This overlap between different dementias may reflect shared pathological mechanisms. The link between frontal WMHs and depression and the absence of occipital WMHs and psychotic symptoms has important implications for understanding the neurobiological basis of these symptoms.

(F Neurol Neurosurg Psychiatry 1999;67:66-72)
Keywords: dementia; white matter hyperintensities; Magnetic resonance imaging

Magnetic resonance imaging can detect hyperintense white matter lesions, especially with proton density and T2 weighted scans. These changes can be divided into those immediately adjacent to the ventricles (periventricular hyperintensities; PVHs) and those located in the deep white matter (white matter hyperintensities; WMHs). Periventricular hyperintensities and WMHs probably result from different pathological processes and have been described in various conditions including normal aging, ${ }^{1}$ vascular dementia, ${ }^{2}$ and Alzheimer's disease. ${ }^{3}$

The prevalence, severity, and distribution of PVHs and WMHs seem to vary between different disorders and with age. Vascular dementia is associated with increased PVHs and WMHs compared with Alzheimer's disease and age matched normal controls. ${ }^{45}$ Patients with Alzheimer's disease also have more extensive PVHs than controls ${ }^{6-10}$ but there are conflicting findings in relation to WMHs. Bowen $e t a l$ found that WMHs were increased in patients with Alzheimer's disease compared with controls, Scheltens $e t a l^{11}$ found an increase only in late onset patients although others have found no difference. ${ }^{10}{ }^{12-14}$ Inconsistency between studies may be attributable to variation in scanning techniques, sample size and selection, and the rating scales used. ${ }^{15}{ }^{16}$

Dementia with Lewy bodies has been recently recognised as a major cause of dementia, ${ }^{17}$ clinically and neuropathologically distinct from Alzheimer's disease and vascular dementia. ${ }^{18}$ It is probably the second commonest form of late life dementia, accounting for up to $20 \%$ of cases. Dementia with Lewy bodies is characterised by the triad of visual hallucinosis, fluctuating cognitive impairment, and parkinsonism. Pathologically, intracytoplasmic, neuronal inclusion bodies (Lewy bodies) are found in the neocortex, limbic cortex, subcortical nuclei, and brainstem. There have been few neuroimaging studies of dementia with Lewy bodies and changes in white matter on MRI in this disease have not previously been investigated.

The clinical importance of changes in white matter seen on MRI in dementia is poorly understood. There is growing evidence that changes in white matter are linked to depressive illness in late life ${ }^{39}$ and possibly psychotic symptoms in late onset schizophrenia. ${ }^{19}{ }^{20}$ 
Whether white matter lesions are involved in the pathogenesis of depressive and psychotic symptoms in dementias is unclear.

The aims of this study were to compare white matter changes on MRI in patients with dementia with Lewy bodies with those with Alzheimer's disease, vascular dementia, and normal age matched controls, and to examine their relation to cognitive and non-cognitive features of dementia. Our hypothesis was that, in common with Alzheimer's disease and vascular dementia, an increased prevalence of changes in white matter would occur in dementia with Lewy bodies. We also tested the hypothesis that changes in white matter in late onset dementias are associated with depressive and possibly psychotic symptoms. A representative sample of community dwelling elderly subjects was included and white matter lesions were rated using a semiquantitative $\operatorname{scale}^{21}$ to record the prevalence, severity, and anatomical distribution of these changes.

\section{Methods}

SELECTION OF PATIENTS WITH DEMENTIA

Eighty patients over the age of 60 years who fulfilled DSM IV ${ }^{22}$ criteria for dementia were recruited. Seventy five patients were obtained from a community dwelling population of patients referred to local old age psychiatry and geriatric medicine services for evaluation of possible dementia. Five patients were recruited from a dementia research clinic. The research was approved by the local ethics committee and all patients, as well as their nearest relative, gave informed consent.

\section{ASSESSMENTS AND DIAGNOSIS}

Diagnosis was made after a detailed clinical assessment. This included an interview with the subject and the most knowledgeable informant using the geriatric mental state/ history aetiology schedule, ${ }^{23}$ review of clinical records, full psychiatric and medical history, and mental state and physical examination. A standard dementia screen was completed which included haematology and biochemistry analysis, thyroid function tests, syphilis serology, B12 and folate concentrations, and CT.

Standardised clinical diagnostic criteria were used to characterise the type of dementia. Diagnoses of Alzheimer's disease, vascular

Table 1 Visual rating of signal hyperintensities

\begin{tabular}{lll}
\hline Lesions & & Score \\
\hline Periventricular hyperintensities (PVH 0-6) & Frontal caps (0-2) & $0=$ absent \\
& Occipital caps (0-2) & $1=\leqslant 5 \mathrm{~mm}$ \\
& Bands: (0-2) & $2=>5 \mathrm{~mm}$ and $<10 \mathrm{~mm}$ \\
White matter hyperintensities (WMH 0-24) & Frontal (0-6) & $0=\mathrm{NA}$ \\
& Parietal (0-6) & $1=<3 \mathrm{~mm} ; n \leqslant 5$ \\
& Occipital (0-6) & $2=<3 \mathrm{~mm} n \geqslant 6$ \\
& Temporal (0-6) & $3=4-10 \mathrm{~mm} n \leqslant 5$ \\
& & $4=4-10 \mathrm{~mm} n \geqslant 6$ \\
& & $5=\geqslant 11 \mathrm{~mm} ; n \geqslant 1$ \\
Basal ganglia hyperintensities (BGH 0-30) & Caudate nucleus (0-6) & As for WMH WM \\
& Putamen (0-6) & \\
& Globus pallidus (0-6) \\
& Thalamus (0-6) & \\
& Internal capsule (0-6) &
\end{tabular}

Semiquantitative rating of signal hyperintensities in separate regions with the range of the scale shown in parentheses: $n=$ number of lesions; $\mathrm{NA}=$ no abnormality. dementia, and dementia with Lewy bodies were made in accordance with NINCDS/ADRDA, ${ }^{24}$ NINDS/AIREN ${ }^{25}$ and dementia with Lewy bodies consensus criteria ${ }^{17}$ respectively by consensus agreement between three experienced raters (JO'B, CB, IMcK). Diagnosis was made blind to MRI findings. Pathological confirmation of clinical diagnosis has since been acquired in four patients. Applying these criteria, 28 patients had Alzheimer's disease (definite $n=2$, probable $n=24$, and possible $n=2), 25$ had vascular dementia (probable $n=15$ and possible $n=10$ ), and 27 had dementia with Lewy bodies (definite $\mathrm{n}=2$, probable $\mathrm{n}=24$, and possible $\mathrm{n}=1$ ).

Within three months of completing MRI all patients underwent a further assessment. Cognitive function was measured using the Cambridge cognitive examination ${ }^{26}$ which incorporates the mini mental state examination (MMSE). ${ }^{27}$ Depressive symptoms were rated using the Montgomery and Asberg depression rating scale (MADRS) ${ }^{28}$ and a history of psychotic symptoms (delusions, misidentifications, auditory hallucinations, and visual hallucinations) since onset of dementia was recorded using the Columbia University scale for psychopathology in Alzheimer's Disease, (CUSPAD) ${ }^{29}$

\section{NORMAL CONTROLS}

Twenty six age matched controls were recruited from among spouses and friends of patients with dementia. A detailed history and examination were undertaken to include demographic data and physical and psychiatric status. All control subjects completed the same assessments as listed above: CAMCOG, MMSE, MADRS, CUSPAD. Exclusion criteria were evidence of current depression (from history or MADRS $>10$ ) or dementia (from history or score $<80$ on the CAMCOG) and history of any other significant neurological, physical, or psychiatric disorder including drug and alcohol misuse.

MRI SCANNING PROTOCOL

All scans were performed on a 1.0 Tesla Siemens Magnetom Impact MRI scanner. Whole brain axial images of $5 \mathrm{~mm}$ thickness (0.5 mm gap) were obtained using proton density weighted and T2 weighted turbo/fast spin echo sequences to allow detailed visualisation of white matter lesions (RARE technique-rapid acquisition with relaxation enhancement: $\mathrm{TR}=2800 \mathrm{~ms}$; TE $14 / 85 \mathrm{~ms}$; matrix $256 \times 256$; field of view $=23 \mathrm{~mm}$ giving pixel size $=0.92 \times 0.92 \mathrm{~mm}$; acquisition time $=4 \mathrm{~min}$ 13s).

WHITE MATTER RATING

Using a standardised scale $^{21}$ white matter lesions were rated from hard copies of proton density and T2 weighted axial images by an experienced rater (PS) blind to diagnosis. The scale provided a semiquantitative measurement of the type, size, frequency, and location of PVHs and WMHs, as shown in table 1. Hyperintensities were rated in five regions: frontal, 
Table 2 Subject characteristics

\begin{tabular}{llllll}
\hline & $\begin{array}{l}D L B \\
(n=27)\end{array}$ & $\begin{array}{l}A D \\
(n=28)\end{array}$ & $\begin{array}{l}\text { VaD } \\
(n=25)\end{array}$ & $\begin{array}{l}\text { Con } \\
(n=26)\end{array}$ & $p$ Value \\
\hline Age (mean (SD)) & $75.9(7)$ & $77.4(5)$ & $76.8(7)$ & $76.2(5)$ & NS \\
Sex (M:F) & $19: 9$ & $10: 18$ & $15: 10$ & $14: 12$ & NS \\
Education (years (SD)) & $9.1(1)$ & $9.7(3)$ & $9.9(1)$ & $10.1(2)$ & NS \\
Length of history (months (SD)) & $38.2(19)$ & $42.4(25)$ & $39.7(25)$ & NS & NS \\
MMSE (mean (SD)) & $13.6(7)$ & $15.5(5)$ & $18.2(4)$ & $28.1(2)$ & $<0.001^{\star}$ \\
CAMCOG (mean (SD)) & $46.1(26)$ & $55.5(16)$ & $62.0(13)$ & $97.2(5)$ & $<0.001^{\star}$ \\
MADRS (mean) & 8.0 & 7.6 & 7.7 & 3.4 & $<0.05$ \\
Delusions (\%) & $18(67)$ & $11(39)$ & $5(20)$ & 0 & $<0.001$ \\
Visual hallucinations (\%) & $23(85)$ & $3(11)$ & $3(12)$ & 0 & $<0.001$ \\
Auditory hallucinations (\%) & $14(52)$ & $4(14)$ & $0(0)$ & 0 & $=0.01$ \\
\hline
\end{tabular}

Con=Controls; $\mathrm{DLB}=$ dementia with Lewy bodies; $\mathrm{AD}=$ Alzheimer's disease; $\mathrm{VaD}=$ vascular dementia.

${ }^{\star}$ post hoc Scheffé test showed significant differences between Con and AD, Con and VaD, Con and DLB $(p<0.001)$, and between VaD and DLB $(p<0.05)$.

Table 3 Periventricular hyperintensities

\begin{tabular}{|c|c|c|c|c|c|}
\hline & $\begin{array}{l}D L B \\
(n=27)\end{array}$ & $\begin{array}{l}A D \\
(n=28)\end{array}$ & $\begin{array}{l}V a D \\
(n=25)\end{array}$ & Con $(n=26)$ & p Value \\
\hline \multicolumn{6}{|l|}{ Bands: } \\
\hline Prevalence & $23(85 \%)$ & $28(100 \%)$ & $24(96 \%)$ & $19(73 \%)$ & \\
\hline Median score & 1 & 1 & 1 & 1 & $<0.05^{\star}$ \\
\hline \multicolumn{6}{|l|}{ Frontal caps: } \\
\hline Prevalence & $25(93 \%)$ & $26(93 \%)$ & $24(96 \%)$ & $23(89 \%)$ & \\
\hline Median score & 1 & 1 & 1 & 1 & NS \\
\hline \multicolumn{6}{|l|}{ Occipital caps: } \\
\hline Prevalence & $22(82 \%)$ & $21(75 \%)$ & $20(80 \%)$ & $8(31 \%)$ & \\
\hline Median score & 1 & 1 & 1 & 0 & $<0.001 \dagger$ \\
\hline \multicolumn{6}{|l|}{ Total PVH: } \\
\hline Prevalence & $27(100 \%)$ & $28(100 \%)$ & $25(100 \%)$ & $24(92 \%)$ & \\
\hline Median score & 3 & 3 & 3 & 2 & $=0.001 \ddagger$ \\
\hline
\end{tabular}

For abbreviations see table 2 .

Post hoc Mann-Whitney $U$ test showed significant differences between:

${ }^{\star} \mathrm{Con}$ and $\mathrm{VaD}(p<0.05)$ and between Con and $\mathrm{AD}(p<0.01)$

$\uparrow \mathrm{Con}$ and $\mathrm{AD}(p=0.001)$ and between Con and VaD, Con and DLB $(p<0.001)$

$\ddagger$ Con and DLB $(p<0.05)$ and between Con and $\mathrm{AD}$, Con and $\mathrm{VaD}(p=0.001)$

temporal, parietal, occipital, and basal ganglia $(\mathrm{BGH})$.

STATISTICAL ANALYSIS

The statistical package SPSS for Windows (Release 7.0) was used for data analysis. ${ }^{30}$ Differences between groups on continuous variables were assessed using analysis of variance (ANOVA) with post hoc Scheffé tests to determine group differences. For non-parametric data a Kruskal-Wallis test was used followed by a post hoc Mann-Whitney $U$ test or Pearson $\chi^{2}$

Table 4 White matter and basal ganglia hyperintensities

\begin{tabular}{|c|c|c|c|c|c|}
\hline & $\begin{array}{l}D L B \\
(n=27)\end{array}$ & $\begin{array}{l}A D \\
(n=28)\end{array}$ & $\begin{array}{l}\mathrm{VaD} \\
(n=25)\end{array}$ & Con $(n=26)$ & $p$ Value \\
\hline \multicolumn{6}{|l|}{ Frontal: } \\
\hline Prevalence & $22(81 \%)$ & $25(89 \%)$ & $24(96 \%)$ & $19(73 \%)$ & \\
\hline Median score & 2 & 2 & 3 & 1 & $<0.05^{\star}$ \\
\hline \multicolumn{6}{|l|}{ Temporal: } \\
\hline Prevalence & $3(11 \%)$ & $4(14 \%)$ & $3(12 \%)$ & $1(4 \%)$ & \\
\hline Median score & 0 & 0 & 0 & 0 & NS \\
\hline \multicolumn{6}{|l|}{ Parietal: } \\
\hline Prevalence & $15(56 \%)$ & $13(46 \%)$ & $19(76 \%)$ & $8(31 \%)$ & \\
\hline Median score & 1 & 0 & 3 & 0 & $<0.01 \dagger$ \\
\hline \multicolumn{6}{|l|}{ Occipital: } \\
\hline Prevalence & $2(7 \%)$ & $3(11 \%)$ & $6(24 \%)$ & $3(12 \%)$ & \\
\hline Median score & 0 & 0 & 0 & 0 & NS \\
\hline \multicolumn{6}{|l|}{ Basal ganglia } \\
\hline prevalence & $7(26 \%)$ & $6(21 \%)$ & $17(68 \%)$ & $3(12 \%)$ & \\
\hline median score & 0 & 0 & 2 & 0 & $<0.001 \ddagger$ \\
\hline \multicolumn{6}{|l|}{ Total } \\
\hline prevalence & $23(85 \%)$ & $25(89 \%)$ & $24(96 \%)$ & $19(73 \%)$ & \\
\hline median score & 3 & 4 & 8 & 1 & $<0.001 \S$ \\
\hline
\end{tabular}

For abbreviations see table 2 .

Post hoc Mann-Whitney $U$ test showed significant differences between:

${ }^{\star} \mathrm{Con}$ and $\mathrm{AD}(p<0.05)$ and between Con and $\mathrm{VaD}(p<0.01)$

†Con and DLB, Con and $\mathrm{AD}(p<0.05)$, and between Con and $\operatorname{VaD}(p<0.001)$

$\ddagger \mathrm{VaD}$ and all other groups $(p<0.001)$

$§ C$ Con and DLB, Con and $\mathrm{AD}, \mathrm{VaD}$ and $\mathrm{DLB}(p<0.05)$, between $\mathrm{VaD}$ and $\mathrm{AD}(p<0.01)$, and between $\mathrm{VaD}$ and $\mathrm{Con}(p<0.001)$ statistic as appropriate, with Fisher's exact probability test calculated for $2 \times 2$ tables when the expected cell frequency was $<5$. Associations between white matter lesions and clinical variables were examined using the MannWhitney $U$ test or Spearman's rank order correlation coefficient $(r)$ as appropriate. All statistical tests were two tailed and were regarded as significant at $\mathrm{p}<0.01$ for correlations and $p<0.05$ for all other tests.

\section{Results}

SUBJECT CHARACTERISTICS

Subject characteristics are summarised in table 2. Groups were well matched for age, sex, history, and years of education. As would be expected, CAMCOG and MMSE scores were significantly lower in all dementia groups compared with controls $(p<0.001)$. Patients with dementia with Lewy bodies were significantly more impaired than those with vascular dementia on MMSE (13.6 $v 18.2 ; \mathrm{p}<0.05)$ and CAMCOG (46.1 v 62.0; $\mathrm{p}<0.05)$.

There were no differences between dementia groups for MADRS scores although all dementia groups had significantly more depressive symptoms than controls $(\mathrm{p}<0.05)$. Patients with dementia with Lewy bodies experienced significantly more delusions $(\mathrm{p}<0.001)$, visual hallucinations $(\mathrm{p}<0.001)$, and auditory hallucinations $(p=0.01)$ than those with Alzheimer's disease and vascular dementia.

\section{PERIVENTRICULAR HYPERINTENSITIES}

The results for PVHs are summarised in table 3. All dementia groups had significantly higher total PVH scores (sum of bands and caps) compared with controls $(p=0.001)$. There were no significant differences between dementia with Lewy bodies, Alzheimer's disease, and vascular dementia, and overall PVHs were present in all dementia patients (100\%) and nearly all age matched controls (92\%).

Analysing bands and caps separately, periventricular bands were significantly more severe in patients with Alzheimer's disease $(p<0.01)$ and vascular dementia $(\mathrm{p}<0.05)$ than in controls, and occipital caps were more severe $(\mathrm{p}<0.001)$ and frequent in all dementia groups compared with controls (dementia with Lewy bodies $82 \%$, vascular dementia $80 \%$, Alzheimer's disease $75 \%$ v controls 31\%; $\mathrm{p}=0.001)$. By contrast, frontal caps were common to all groups (vascular dementia 96\%, Alzheimer's disease 93\%, dementia with Lewy bodies $92 \%$, controls $89 \%$ ).

Periventricular hyperintensities were positively correlated with age in all patients (total PVH score $r=0.41, \mathrm{p}<0.001)$ but were not associated with cognitive impairment or depressive and psychotic symptoms.

\section{WHITE MATTER AND BASAL GANGLIA} HYPERINTENSITIES

The results for WMHs and basal ganglia lesions are presented in table 4. Photographs of MRI demonstrating white matter changes in subjects from each group are shown in the figure. 

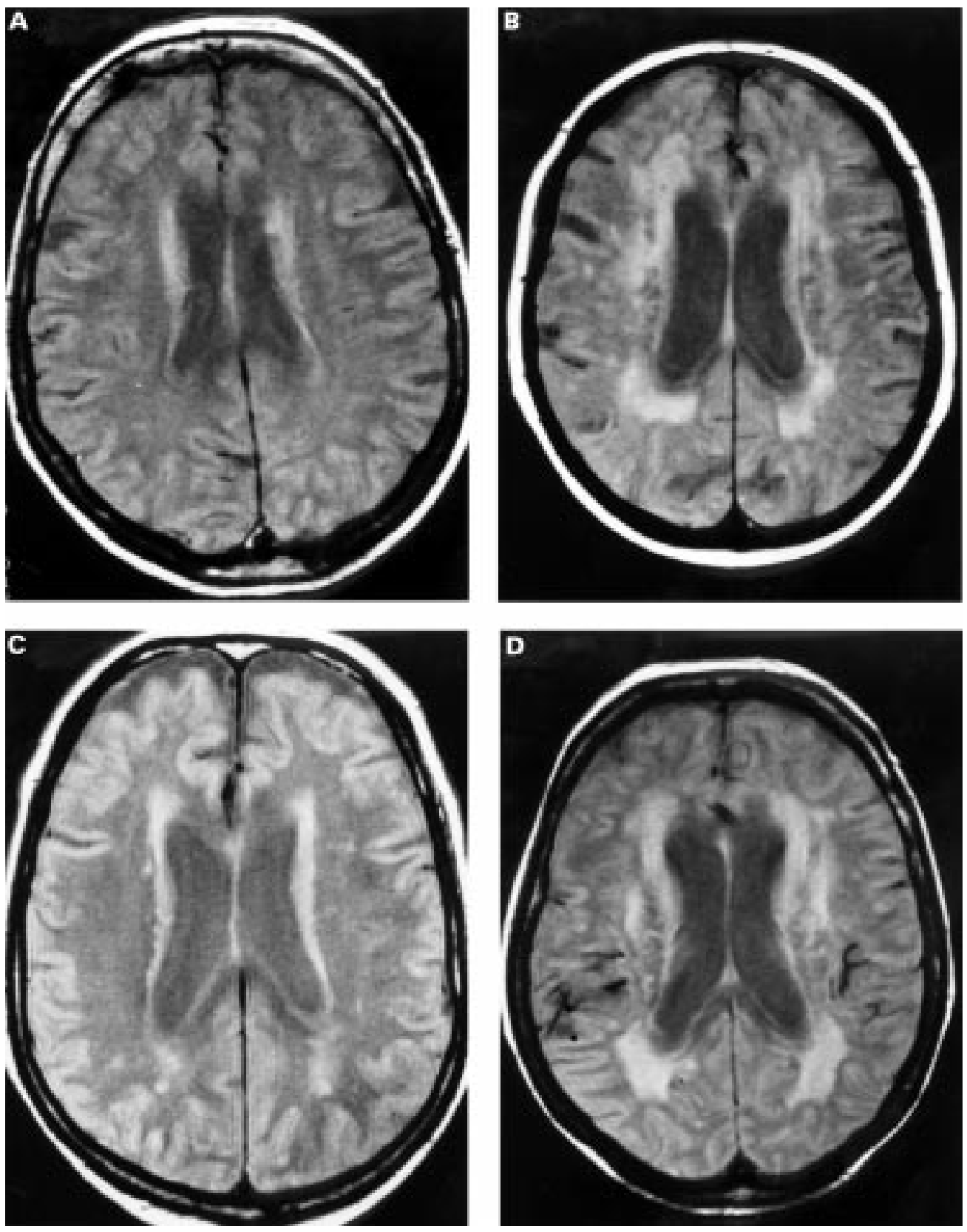

Axial proton density MRI showing white matter lesions and ratings: $(A)$ normal control $(P V H$ score $=0 ; W M H$ score $=0)$; (B) patient with vascular dementia (PVH: frontal caps $=2$, occipital caps $=2 ; W M H$ frontal $=5$, parietal $=6)$; $(C)$ patient with pathologically confirmed dementia with Lewy bodies (PVH: frontal caps=2, occipital caps=1; WMH frontal=2, parietal=3); (D) patient with pathologically confirmed Alzheimer's disease bodies (PVH: frontal caps=2, occipital caps $=2$, WMH frontal=6, parietal=6).

Total deep hyperintensity scores (sum of the frontal, temporal, parietal, occipital, and basal ganglia scores) were significantly higher in all dementia groups compared with controls (vascuylar dementia median score $=8 v$ control median score $=1, \mathrm{p}<0.001$; dementia with Lewy bodies median score $=3$, and Alzheimer's disease median score $=4 v$ control median score, $\mathrm{p}<0.05)$. Comparing the dementia groups, patients with vascular dementia had higher total lesion scores than those with Alzheimer's disease $(p<0.01)$ and dementia with Lewy bodies $(\mathrm{p}<0.05)$. Overall, basal ganglia lesions were specifically associated with vascular dementia compared with all other groups (vascular dementia $68 \%$, dementia with
Lewy bodies $26 \%$, Alzheimer's disease $21 \%$, controls $12 \%$; $<<0.001$ ).

A link between regional changes in white matter and specific non-cognitive symptoms in dementia patients was seen. The presence of frontal WMHs was associated with higher depression scores (mean MADRS score for patients with frontal WMHs $=8.3 v$ without $=3.4 ; \mathrm{p}<0.05)$. The presence of delusions $(p<0.01)$ and visual hallucinations $(p<0.05)$ was associated with the absence of occipital WMHs. No patient with delusions $(n=34)$ had WMHs, and only one patient with visual hallucinations $(n=29)$ had occipital WMHs. Auditory hallucinations showed a similar trend $(p=0.06)$. White matter hyperintensity scores 
did not correlate with age or cognitive impairment.

\section{Discussion}

This is the first study to examine the presence of changes in white matter on MRI in dementia with Lewy bodies. The inclusion of patients with Alzheimer's disease and patients with vascular dementia allowed comparison of changes in white matter between the three major types of late life dementia. Periventricular hyperintensities and WMHs were found to occur often in patients with dementia with Lewy bodies, Alzheimer's disease, vascular dementia, and aged matched normal controls. These changes were, however, significantly more extensive in the dementia groups compared with controls.

The major strengths of this study include the recruitment of a representative community sample of elderly patients with late life dementia, well matched for age, sex, education, and duration of illness. Brain MRI was acquired using a standardised protocol, and changes in white matter were assessed blind by an experienced rater using a validated scale which provided detailed information on the frequency, size, and location of lesions. A potential criticism of this study is the reliance on clinical, rather than pathological, diagnoses. However, clinical diagnosis were made after detailed assessments and in accordance with standardised criteria. In the case of dementia with Lewy bodies, we have shown that the clinical criteria have a high specificity (0.95) indicating few false positive clinical diagnoses. ${ }^{31}$ A recent clinicopathological validation of current clinical criteria for dementias found a similar level of specificity (1.00), but low sensitivity (0.22), for the consensus diagnosis of probable dementia with Lewy bodies. ${ }^{32}$ Overall the clinical criteria seem to have a high positive predictive value for pathology of dementia with Lewy bodies itself, although the diagnosis does not exclude the possibility of mixed disease. ${ }^{32}$ The profile of patients with dementia with Lewy bodies studied conformed well with other published cohorts. ${ }^{33}$ Pathological confirmation of diagnosis has since been acquired in four patients (dementia with Lewy bodies $n=2$; Alzheimer's disease $n=2$ ).

PERIVENTRICULAR HYPERINTENSITIES

Periventricular hyperintensities were found in all patients with dementia and $92 \%$ of controls. Previous reports have found PVHs to occur in $44 \%$ to $100 \%$ of patients with Alzheimer's disease, $70 \%$ to $100 \%$ with vascular dementia, and $8 \%$ to $92 \%$ of elderly controls. ${ }^{1-3}$ In common with other studies, ${ }^{91134-36}$ a positive correlation between the PVHs and age was also found. The high prevalence of PVHs in this study may therefore reflect, in part, the age of patients.

As found previously, PVHs were more severe in dementia patients than in controls. ${ }^{6} 91137$ However, not all PVHs were equal in this regard. Bands and occipital caps, by contrast with frontal caps, were more severe and common in dementia groups than in controls.
The near ubiquitous presence of frontal caps lends further support to the notion that they occur as a normal, age related change. ${ }^{38} 39$ Histologically, frontal PVHs probably result from areas of demyelination and astrocytic gliosis. ${ }^{40}$

Divergence in the severity of periventricular bands and occipital caps between demented and non-demented patients suggests that pathological processes contribute to their development. The convergence of results within the dementia groups would argue in favour of a common pathogenesis. Interestingly, Fazekas et $a l^{10}$ found that patients with Alzheimer's disease had significantly more PVHs than controls but that this association was not independently linked to the disease; rather it seemed to be related to atrophy as measured by ventricular enlargement. Other studies have also found an association of PVHs and atrophy, ${ }^{41}$ and in vitro pathological studies indicate that PVHs are associated with loss of the ependymal lining, demyelination, and gliosis. ${ }^{42-44}$ Damage to the integrity of the ependymal lining may in turn result in abnormal dynamics of CSF and increased water content. ${ }^{344243}$ Histological studies of PVHs in dementia with Lewy bodies are necessary to establish whether similar changes occur.

The absence of a link between PVHs and the clinical features of dementia with Lewy bodies was consistent with negative reports involving non-demented elderly patients, patients with Alzheimer's disease, and patients with vascular dementia. ${ }^{2}$ The relation between PVHs seen on MRI and clinical features remains unclear. Perhaps, as some authors have suggested, MRI is more sensitive at detecting PVHs, but CT is more specific and detects lesions which are more tightly coupled with pathological change and outcome. ${ }^{45}$

WHITE MATTER AND BASAL GANGLIA

HYPERINTENSITIES

White matter hyperintensities also occurred often in all groups and differences between demented and non-demented patients largely resulted from variations in the severity and distribution of lesions. The pattern of WMHs in dementia with Lewy bodies was broadly similar to Alzheimer's disease, but less extensive than vascular dementia. The prevalence of WMHs in vascular dementia and Alzheimer's disease conformed well with previous studies, which have detected WMHs in up to $100 \%$ of patients with vascular dementia ${ }^{72}$ and between $60 \%-100 \%$ of patients with Alzheimer's disease. $^{2}$

The pathological correlates of WMHs in dementia with Lewy bodies have not yet been determined. The similar pattern of hyperintensities in dementia with Lewy bodies and Alzheimer's disease suggests that they may share a convergence in white matter pathology. White matter lesions in Alzheimer's disease have been linked to ischaemic changes ${ }^{46}$ but histological findings from other studies have identified various abnormalities, including dilated perivascular spaces, arteriosclerosis, partial loss of myelin and axons, gliosis, lacunar 
infarcts, and congenital diverticula. ${ }^{3}$ Clearly not all WMHs result from ischaemic damage and postmortem examination, ideally preceded by postmortem brain MRI, is necessary to determine the nature of white matter pathology in dementia with Lewy bodies. In vivo characterisation of WMHs using proton magnetic resonance spectroscopy may also provide useful pathological and diagnostic information. ${ }^{47}$

The pronounced increase of basal ganglia lesions in vascular dementia is consistent with previous reports ${ }^{5124849}$ and suggests that these changes may be useful as a diagnostic aid in the differential diagnosis of vascular dementia from dementia with Lewy bodies as well as Alzheimer's disease. ${ }^{12}$ Interestingly, the lack of an association between dementia with Lewy bodies and basal ganglia hyperintensities indicates that these lesions are unlikely to contribute to the development of parkinsonism in dementia with Lewy bodies.

There are contrasting reports on the association between changes in white matter and cognitive function. ${ }^{34}$ Discrepancies may be explained by methodological differences. In common with many other studies, the rating of white matter lesions in this study was not volumetric. Semiquantitative rating scales may not be sufficiently sensitive to detect correlations with cognition unless sample size is large.$^{50} \mathrm{By}$ contrast, positive correlations have been reported with moderate sample sizes using volumetric measures. ${ }^{5152}$ In addition, in healthy patients a "threshold" effect has been reported such that cognitive deficits only become evident beyond a certain severity of changes in white matter. ${ }^{51}$ Longitudinal studies might provide further insights in to the nature of any interaction between WMHs, cognitive function, and disease progression.

Non-cognitive features of dementias occur commonly and are clinically important. There is growing evidence that frontal and subcortical WMHs are implicated in late life depression and influence outcome, possibly by disruption of frontal-subcortical circuits. ${ }^{93-56}$ The findings of this study suggest that frontal WMHs may also be relevant in understanding depression in dementia, and imply a common pathophysiology of depression irrespective of diagnosis. ${ }^{52}$

The association of psychotic symptoms with specific brain abnormalities in dementias remains controversial. Howanitz et $a \bar{l}^{7}$ studied patients with Alzheimer's disease and found that hallucinations were associated with cerebral atrophy and enlarged third ventricles but not abnormalities in white matter, though they did not examine regional changes in white matter. In this study, there was a negative association between occipital WMHs and psychosis which was not explained by group differences between dementia with Lewy bodies (who hallucinate a lot) and other groups (who hallucinate less). This findings could imply that a "disease" free occipital lobe, presumably with abnormalities located in other brain regions, is necessary to develop psychosis, and that disruption of neural circuits involving visual association areas might prevent hallucinations. Using functional MRI Howard et $a l^{8}$ have suggested that visual hallucinations are, at least in part, located in the primary visual cortex, raising the possibility that strategically located lesions in white matter may disrupt this process. Whether occipital WMHs might suppress visual hallucinations is clearly an area which requires further investigation.

\section{Conclusion}

Periventricular hyperintensities were found to be common in dementia with Lewy bodies as well as Alzheimer's disease, vascular dementia, and aged matched controls, but were uniformly more severe in all major types of dementia compared with controls. This could indicate that PVHs result from a common pathogenesis, possibly related to brain atrophy. The pattern of deep hyperintensities in dementia with Lewy bodies was broadly similar to Alzheimer's disease and both groups showed modest increases in the severity of changes in white matter compared with controls. Overall WMHs and basal ganglia lesions were most pronounced in patients with vascular dementia. A link between the presence of frontal WMHs and depression and the absence of occipital WMHs and psychotic symptoms was found. This has important implications for understanding the neurobiological basis of these symptoms.

We thank Mr P English for producing the magnetic resonance images, and Mr A Gray, Ms K Lowry, and Ms D Roberts for facilitating the scanning of patients. The research was supported facilitating the scanning of patients. The research was supported by a grant from
Authority, UK.

1 Meyer JS, Kawamura J, Terayama Y. White matter lesions in the elderly. 7 Neurol Sci 1992;110:1-7.

2 Mirsen TR, Merskey H. Leukoaraiosis. In: Burns A, Levy R, eds. Dementia. London: Chapman and Hall, 1994:641-52.

3 O'Brien JT, Ames D, Schwietzer I. White matter in depression and Alzheimer's disease: a review of magnetic resonance imaging studies. International fournal of Geriatric resonance imaging studies.
Psychiatry 1996;11:681-94.

4 Almkvist O, Wahlund LO, Andersson-Lundman G, et al. White-matter hyperintensity and neuropsychological functions in dementia and healthy aging. Arch Neurol 1992;49: 626-32.

5 Schmidt R. Comparison of magnetic resonance imaging in Alzheimer's disease, vascular dementia and normal aging. Eur Neurol 1992;32:164-9

6 Fazekas F, Chawluk JB, Alavi A, et al. MR signal abnormalities at 1.5T in Azheimer's disease and normal ageing. Am $\mathcal{f}$ Roentgenol 1987;149:351-6.

7 Bowen BC, Barker WW, Loewenstein DA, et al. MR signal abnormalities in memory disorder and dementia. AfR Am f Roent genol 1990;154:1285-92.

8 Mirsen TR, Lee DH, Wong CJ, et al. Clinical correlates of white matter changes on magnetic resonance imaging scans of the brain. Arch Neurol 1991;48:1015-21.

9 O'Brien JT, Desmond P, Ames D, et al. A magnetic resonance imaging study of white matter lesions in depression and Alzheimer's disease. Br f Psychiatry 1996;168: sion and

10 Fazekas F, Kapeller P, Schmidt R, et al. The relation of cerebral magnetic resonance signal hyperintensities to Alzheimer's disease. F Neurol Sci 1996;142:121-5.

11 Scheltens P, Barkhof F, Valk J, et al. White matter lesions on magnetic resonance imaging in clinically diagnosed Alzheimer's disease. Brain 1992;115:735-48

12 Erkinjuntti T, Ketonen L, Sulkava R, et al. Do white matter changes on MRI and CT differentiate vascular dementia from Alzheimer's disease? $\mathcal{F}$ Neurol Neurosurg Psychiatry 1987;50:37-42.

13 Leys D, Soetaert G, Petit H, et al. Periventricular and white matter magnetic resonance imaging hyperintensities do not differ between Alzheimer's disease and normal aging. Arch Neurol 1990;47:524-7.

14 Miller DS, Kumar A, Yousem DM, et al. MRI high-intensity signals in late-life depression and Alzheimer's disease. American fournal of Geriatric Psychiatry 1994;2:332-7.

15 Mantyla R, Erkinjuntti T, Salonen O, et al. Variable agreement between visual rating scales for white matter hyperintensities on MRI. Comparison of 13 rating scales in a poststroke cohort. Stroke 1997;28:1614-23. 
16 Scheltens $\mathrm{P}$, Erkinjunti $\mathrm{T}$, Leys $\mathrm{D}$, et al. White matter changes on CT and MRI: an overview of visual rating scales. European Task Force on age-
changes. Eur Neurol 1998;39:80-9.

17 McKeith IG, Galasko D, Kosaka K. Consensus guidelines for the clinical and pathological diagnosis of dementia with Lewy bodies (DLB): report of the consortium on DLB international workshop. Neurology 1996;47:1113-24.

18 Perry RH, Irving D, Blessed G, et al. Clinically and neuropathologically distinct form of dementia in the elderly. Lancet 1989;i:166.

19 Miller BL, Lesser IM, Boone KB, et al. Brain white-matter lesions and psychosis. Br F Psychiatry 1989;155:73-8.

20 Miller BL, Lesser IM, Boone KB, et al. Brain lesions and cognitive function in late-life psychosis. $\mathrm{Br}$ f Psychiatry 1991;158:76-82.

21 Scheltens P, Barkhof F, Leys D, et al. A semiquantitative rating scale for the assessment of signal hyperintensities on ing scale for the assessment of signal hyperintensities on

22 American Psychiatric Association. Diagnostic and statistical manual of mental disorders. 4th ed. Washington, DC: APA 1994.

23 Copeland JRM, Kelleher MJ, Kellett JM, et al. A semistructured clinical interview for the assessment, diagnosis and mental state in the elderly: the geriatric mental state schedule. Psychol Med 1976;6:439-49.

24 McKhann G, Drachman D, Folstein M, et al. Clinical diagnosis of Alzheimer's disease: report of the NINCDSADRDA Work Group under the auspices of Department of Health and Human Service Task Force on Alzheimer's disease. Neurology 1984;34:939-44

25 Roman GC, Tatemischi T, Erkinjuntti T, et al. Vascular dementia: diagnostic criteria for research studies. Report of the NINDS-AIRENS international workshop. Neurology 1993;43:250-60.

26 Roth M, Tym E, Mountjoy C, et al. CAMDEX. A standardised instrument for the diagnosis of mental disorder in the elderly with special reference to the early detection of elderly with special reference to the early
dementia. Br f Psychiatry 1986;149:698-709.

27 Folstein M, Folstein S, McHugh P. "Mini-mental state." A practical method for grading the cognitive state of patients practical method for grading the cognitive state

28 Montgomery SA, Asberg M. A new depression scale designed to be sensitive to change. $\mathrm{Br} \mathcal{F}$ Psychiatry 1979;134:382-9.

29 Devanand DP, Miller L, Richards M, et al. The Columbia University scale for psychopathology in Alzheimer's disease. Arch Neurol 1992;49:371-6.

30 Norusis MJ. Statistical package for the social sciences: SPSS for windows version 7.0. Chicago: SPSS, 1995.

31 McKeith IG, Fairbairn AF, Bothwell RA, et al. An evaluation of the predictive validity and inter-rater reliability of clinical diagnostic criteria for senile dementia of Lewy body type. Neurology 1994;44:872-7.

32 Holmes C, Cairns N, Lantos P, et al. Validity of current clinical criteria for Alzheimer's disease, vascular dementia and dementia with Lewy bodies. Br f Psychiatry 1999;174: 45-50.

33 Perry RH, McKeith IG, Perry EK. Dementia with Lewy bodies: clinical, pathological, and treatment issues. Cambridge: Cambridge University Press, 1996.

34 Zimmerman RD, Fleming CA, Lee BC, et al. Periventricular hyperintensities as seen by magnetic resonance: prevalence and significance. Am $\mathcal{F}$ Neuroradiol 1986;7:13-20.

35 Bennett DA, Gilley DW, Wilson RS, et al. Clinical correlates of high signal lesions on magnetic resonance imaging in Alzheimer's disease. I Neurol 1992;239:186-90.

36 Bondareff WD, Raval J, Woo B, et al. Magnetic resonance imaging and the severity of dementia in older adults. Arch Gen Psychiatry 1990;47:47-51.

37 Harrell LE, Duvall E, Folks DG, et al. The relationship of high-intensity signals on magnetic resonance images to cognitive and psychiatric state in Alzheimer's disease. Arch Neurol 1991;48:1136-40.

38 Sze G, De Armond SJ, Brant-Zawadzki M, et al. Foci of MRI signal (pseudo lesions) anterior to the frontal horns: histologic correlations of a normal finding. Am f Neuroradiol 1986;7:381-7.

39 Leifer D, Buonanno FS, Richardson EPJ. Clinicopathologic correlations of cranial magnetic resonance imaging of periventricular white matter. Neurology 1990;40:911-8.

40 Van Swieten JC, Van Den Hout HW, Van Ketel BA, et al. Periventricular lesions in the white matter on magnetic resonance in the elderly. Brain 1991;114:761-74.

41 Waldemar G, Christiansen P, Larsson HBW, et al. White matter resonance hyperintensities in dementia of the Alzheimer type: morphological and regional cerebral blood flow correlates. 7 Neurol Neurosurg Psychiatry 1994;57: $1458-65$.

42 Chimowitz MI, Estes ML, Furlan AJ, et al. Further observations on the pathology of subcortical lesions identified on magnetic resonance imaging. Arch Neurol 1992;49:747-52.

43 Fazekas F, Kleimert R, Offenbacher H, et al. Pathologic correlates of incidential MRI white matter hyperintensities. Neurology 1993;43:1683-9.

44 Scheltens P, Barkhof F, Leys D, et al. Histopathologic correlates of white matter changes on MRI in Alzheimer's disease and normal aging. Neurology 1995;45:883-8.

45 Skoog I. Risk factors for vascular dementia: a review. Dementia and Geriatric Cognitive Disorders 1994;5:137-44.

46 Englund E, Brun A. White matter changes in dementia of Alzheimer's type: the difference in vulnerability between cell compartments. Histopathology 1990;16:433-9.

47 Kattapong VJ, Brooks WM, Wesley MH, et al. Proton magnetic resonance spectroscopy of vascular- and Alzheimertype dementia. Arch Neurol 1996;53:678-80.

48 Duara R, Barker W, Loewenstein D, et al. Sensitivity and specificity of positron emission tomography and magnetic resonance imaging studies in Alzheimer's disease and multi-infarct dementia. Eur Neurol 1989;29(suppl 3):9-15.

49 Wahlund LO. Brain imaging and vascular dementia. Dement Geriatr Cogn Disord 1994;5:193-6.

50 Longstreth WJ. Brain abnormalities in the elderly: frequency and predictors in the United States (the cardiovascular health study). Cardiovascular Health Study Collaborative Research Group. Fournal Neurological Transmission SuppleResearch Group. Four

51 Boone KB, Miller BL, Lesser IM, et al. Neuropsychological correlates of white-matter lesions in healthy elderly subjects. A threshold effect. Arch Neurol 1992;49:549-54.

52 DeCarli C, Murphy DG, Tranh M, et al. The effect of white matter hyperintensity volume on brain structure, cognitive performance, and cerebral metabolism of glucose in 51 healthy controls. Neurology 1995;45:2077-84

53 Salloway S, Malloy P, Kohn R, et al. MRI and neuropsychoogical differences in early- and late-life-onset geriatric depression. Neurology 1996;46:1567-74.

54 Hickie I, Scott E, Wilhelm K, et al. Subcortical hyperintensities on magnetic resonance imaging in patients with severe depression. Biol Psychiatry 1997;42:367-74.

55 Greenwald BS, Kramer-Ginsberg E, Krishnan KR, et al. Neuroanatomic localization of magnetic resonance imaging signal hyperintensities in geriatric depression. Stroke 1998;29:613-17.

56 Hirono N, Mori E, Ishii K, et al. Frontal lobe hypometabolism and depression in Alzheimer's disease. Neurology 1998;50:380-3.

57 Howanitz E, Bajulaiye R, Losonczy M. Magnetic resonance imaging correlates of psychosis in Alzheimer's disease. $\mathcal{F}$ Nerv Ment Dis 1995;183:548-9.

58 Howard R, David A, Woodruff P, et al. Seeing visual hallucinations with functional magnetic resonance imaging. Dement Geriatr Cogn Disord 1997;8:73-7. 